\title{
El Programa Institucional de Tutorías (PIT): impacto en la formación de los estudiantes universitarios
}

\section{The Institutional Tutoring Program (PIT): impact on the training of university students}

CEPEDA-GONZALEZ, María Cristina*†, VILLARREAL-SOTO, Blanca Margarita, RAMOSJAUBERT, Roció Isabel, GARCIA-CONTRERAS, Laura Patricia y MENDOZA-BELMARES, Abraham

Universidad Autónoma de Coahuila. Boulevard Venustiano Carranza esquina con González Lobo S/N

ID $1^{\text {er }}$ Autor: María Cristina, Cepeda-Gonzalez / ORC ID: 0000-0003-0676-2412, Researcher ID: 1651-2018, CVU CONACYT ID: 567204

ID $1^{\text {er }}$ Coautor: Blanca Margarita, Villarreal-Soto / ORC ID: https: 0000-0001-9314-8001, Researcher ID:2357-2018, CVU CONACYT ID: 947979

ID $2^{\text {do }}$ Coautor: Roció Isabel, Ramos-Jaubert / ORC ID: 0000-0003-3289-5390, Researcher ID: T-1652-2018, CVU CONACYT ID: 152250

ID $3^{\text {er }}$ Coautor: Laura Patricia, Garcia-Contreras / ORC ID: 0000-0002 9020 3762, CVU CONACYT ID: 445949

ID $4^{\text {to }}$ Coautor: Ahraham, Mendoza-Belmares / ORC ID: 0000-0003-4659-998X, CVU CONACYT ID: 1016215

DOI: $10.35429 / J U P .2019 .8 .3 .1 .6$

Recibido: 15 de Abril, 2019; Aceptado 20 de Junio, 2019

\begin{abstract}
Resumen
El presente estudio tiene como objetivo general analizar la relación que existe entre el programa institucional de tutorías y la formación de los estudiantes universitarios. Es un estudio de tipo exploratorio, se examina el fenómeno desde una diferente perspectiva a otros estudios realizados; descriptiva, puesto que se especifica como es el fenómeno de estudio y correlacional, explica la relación entre los factores que integran el fenómeno de estudio (Hernández, 2014). Se aplica una encuesta a 100 alumnos de la Facultad de Ciencia, Educación y Humanidades, de la Universidad Autónoma de Coahuila, los resultados son explorados en los siguientes análisis: Descriptivo, internacional y relacional. Los principales resultados son que el apoyo de servicios personalizado de tutorías tiene impacto en la formación de los estudiantes desde el desarrollo de habilidades como la toma de decisiones y en sus recursos de aprendizaje. Además en su cumplimiento de objetivos planteados. A partir de los resultados se pretende mejorar el trabajo tutorial desde la perspectiva del estudiante que les permita un cambio cultural en cuanto a cómo llevar el proceso de la tutoría.
\end{abstract}

Tutorías, Impacto, Formación

\begin{abstract}
The present study has as main objective to analyze the relationship between the mentoring institutional program and the formation of college students. It is an exploratory study type, phenomenon is examined from a distinct perspective to other studies made; descriptive, since it is specified how is the phenomenon study and correlational (Hernandez 2014). A survey is applied to 100 students from the Science, Education and Humanity faculty of the Autonomous University of Coahuila, results are explored in the following analysis; Descriptive, integrational and relational. Main results are that support of the personalized services of mentoring have impact in the student's formation since the skills development as decision taking and in their learning resources. Besides in their achievement of planed objectives. Parting from the results is pretended to improve the mentoring work from the student's perspective that allows them a cultural change as to how to take the process of tutoring.
\end{abstract}

Mentoring, Impact, Forma

Citación: CEPEDA-GONZALEZ, María Cristina, VILLARREAL-SOTO, Blanca Margarita, RAMOS-JAUBERT, Roció Isabel, GARCIA-CONTRERAS, Laura Patricia y MENDOZA-BELMARES, Abraham. El Programa Institucional de Tutorías (PIT): impacto en la formación de los estudiantes universitarios. Revista de Políticas Universitarias. 2019. 3-8: 1-6.

\footnotetext{
* Correspondencia del Autor (Correo electrónico: c_cepeda39@ hotmail.com)

$\dagger$ Investigador contribuyendo como primer autor.
} 


\section{Introducción}

En México, la Asociación Nacional de Instituciones de Educación Superior (ANUIES) formuló, como uno de los programas estratégicos para el desarrollo del sistema de ES, el programa de desarrollo integral de estudiantes. Dentro de este programa, se formula la tutoría como una de las herramientas institucionales orientadas al mejoramiento de la calidad de dicho nivel formativo. Como resultados, surgen los programas institucionales de tutoría. Así, la Secretaría de Educación Pública introduce las políticas de tutoría dentro de los planes nacionales de la ES, imponiendo la obligatoriedad de la tutoría de modo particular.

La misma ANUIES impulsa a las instituciones de ES, particularmente a las de carácter público, para que pongan en marcha sistemas de tutoría, por medio de los cuales los alumnos cuenten, a lo largo de toda su formación, con el consejo y el apoyo de un profesor debidamente preparado. La ANUIES la define como el «[...] acompañamiento y apoyo docente de carácter individual, ofrecido a los estudiantes como una actividad más de su currículum formativo, que puede ser la palanca que sirva para una transformación cualitativa del proceso educativo en el nivel superior» (ANUIES, 2001: 21). El programa de tutoría apoya el logro de una formación integral en los universitarios, al efectuar acciones que permitan atender y formar a los estudiantes en los aspectos que inciden en su maduración personal: conocimientos, actitudes, habilidades, valores, sentido de justicia y desarrollo emocional y ético (ANUIES, 2001).

A partir de tales directrices, la tutoría cobró relevancia en la instituciones de ES, de modo que, a los pocos años, la mayoría de las universidades públicas contaban con programas institucionales de tutorías (Romo López y Romero Herrera, 2015).

Por su parte la Universidad Autónoma de Coahuila implementó en mayo del 2010 el Programa Institucional de Tutorías (PIT) como una estrategia de prevención y atención a estudiantes de los niveles medio superior y superior, con la finalidad de abatir el rezago educativo, deserción y reprobación de dichos educandos.
Las instituciones de educación superior coinciden en que la atención personalizada a los estudiantes puede ayudar a disminuir los índices de reprobación y rezago escolar, a reducir las tasas de abandono de los estudios y a mejorar la eficiencia terminal, así mismo concuerdan en la tutoría como una estrategia viable para promover el mejoramiento de la calidad de la educación superior (Serna \& Cruces, 2004).

\section{Problemática Atendida}

Por lo anterior en el presente estudio su interrogante principal es: ¿Qué relación existe entre el programa institucional de tutorías y la formación de los estudiantes universitarios?

\section{Desarrollo}

\section{Tutor}

El trabajo primordial del tutor es buscar que los alumnos se encuentren gratificados en la relación tutorial lo cual no ha de suponer la ausencia de esfuerzo por parte de los tutorados sino que implica que el esfuerzo del alumnado ha de estar presente, y es preciso que sea bien asumido. Encontrarse a gusto, pero estimulado en la dinámica tutoral por las propuestas compartidas; lo anterior resulta imprescindible para conseguir mejores rendimientos. (Adell. 2002) Young y Wringht, (2001) comentan que el "tutor es una persona hábil, cuenta con información, es dinámico y está comprometido en mejorar las habilidades de otro individuo. Los tutores entrenan, enseñan y modelan a los tutorados". Por su parte Ragins (1997) dice que "los tutores son individuos con experiencia, conocimiento y compromiso para proveer soporte y movilidad a las carreras de sus tutorados".

\section{Tutoría}

En relación a la tutoría, que incluye la impartición de cursos Coughlan, T., Pitt, R., \& Farrow, R. (2019) realizaron un proyecto de los diversos usos e impactos de los cursos abiertos producidos a través de una iniciativa internacional Recursos Educativos Abiertos (REA) donde se presenta un análisis temático de entrevistas retrospectivas y documentación de este estudio de caso, guiado por una revisión de conceptos relevantes de la innovación y la literatura REA. 
Fullerton (1996) señala que el concepto de tutoría es complicado, ya que existen varias definiciones y el fenómeno parece no estar organizado. Hay conceptos que son relacionados con tutoría, por ejemplo: dar clases privadas, supervisión, entrenamiento, consejería, etc., que si bien tienen que ver con aspectos de la tutoría, por sí solos no le dan significado.

En este sentido para Álvarez (2014) la tutoría universitaria se significa como una función de acompañamiento, de orientación y de apoyo al alumnado en su proceso de personalización de los aprendizajes y del desarrollo de las competencias, tanto a nivel personal como profesional, a lo largo de su trayectoria, con el horizonte dinámico del proyecto de vida, un proyecto personal y profesional.

Ahora bien, la tutoría universitaria se configura como un proceso estructurado técnicamente, con el fin de ofrecer a los estudiantes la información y la formación necesarias para el desarrollo de su carrera y de facilitar su inserción en la sociedad como ciudadanos y profesionales activos. Para ser efectiva y eficaz, la tutoría debe incardinarse en el proceso de enseñanza-aprendizaje, ya que implica un aspecto gradual y acumulativo de experiencias que tienen para cada alumno su propia significación personal (Sancho Sora, Baillo Falo, Vicente Serrano y Larumbe Orraitz, 2006; Martínez Clares, Pérez Cusó y Martínez Juárez, 2014; Álvarez, 2014)

Para Bey (1995) entre la variedad de interpretaciones, hay una vaguedad y una falta de claridad sobre antecedentes, resultados, características y mediadores en las relaciones de tutoría. Sin embargo, el tema común a través de la mayoría de las definiciones de tutoría es que se identifica como una relación entre dos individuos, uno con alto nivel de pericia en un particular setting, o área práctica, y otro con menor habilidad y conocimientos en la comunidad, profesión u organización. A partir de esta premisa los autores agregan otros factores importantes, por ejemplo: la consistencia y el periodo de tiempo, competencias específicas a desarrollar, potencial de ayuda y desarrollo de la carrera de un miembro más joven.
La tutoría académica se propone como una opción para reducir los índices de deserción y reprobación (Waldo, 2002), hace más de 10 años que se pensaba que de esta manera al tener tutorados los alumnos disminuirían la deserción escolar y junto con ello la reprobación, hoy en día se pretende seguir estudiando este fenómeno dado a que en las universidades sigue habiendo deserción, buscando la manera de ver cómo influyen las tutorías en ello.

Sin embargo, Para Cruz et al. (2008) la tutoría se ocupa de atender problemas relacionados con la eficiencia terminal, reprobación, habilidades de estudio, dificultades de aprendizaje, ansiedad ante los exámenes, estabilidad emocional, actitudes hacia la profesión y opciones de trayectoria, entre otros.

De acuerdo a Li, et al. (2019) En la interacción entre padres y maestros cuando existe, puede tener efectos positivos en el aumento del rendimiento académico.

\section{Tutorado}

Es todo estudiante, quien recibe orientación, consejería, dirección, tutoría informativa y asesoría y seguimiento de un proceso académica y socio afectivo a través de un tutor a partir de su ingreso hasta su egreso. (Adams, 1993) Señala como atributos de los tutorados: responsabilidad, iniciativa, ingeniosidad, habilidad para desarrollar un plan a fin de alcanzar sus metas y escuchar los consejos del tutor, además de no asumir el rol de niño necesitado a expensas de lo que disponga el tutor. Sin embargo, el desarrollo de la relación Tutor-Tutorado implica el aprendizaje cooperativo que también se extiende a los demás escolares en situación de pares, y suele contribuir a la formación sociomoral (Escámez y Sales, 2002) y (SEP, 2003, pág. 121).

\section{Metodología}

Se trata de un estudio de tipo exploratorio, ya que se examina el fenómeno desde diferentes perspectivas a otros estudios realizados; descriptiva, porque se detalla y especifica como es el fenómeno de estudio y correlacional, debido a que busca explicar la relación entre los factores que integran el fenómeno de estudio. (Hernández, 2014). 
El presente trabajo es un análisis al interior de la Facultad de Ciencia, Educación y Humanidades, se diseña una encuesta de 140 variables que son medidas con una escala decimal y es aplicada a 100 alumnos distribuidos en 4 semestres. Se efectúa la prueba piloto que permite hacer adecuaciones a los reactivos, en la aplicación definitiva del cuestionario se obtiene un alpha de Cronbach de .95. Se exploran los resultados en tres niveles de la estadística: Descriptiva desde frecuencias y porcentajes, Integracional con el análisis factorial y la Relacional con el análisis de regresión.

\section{Objetivo general}

Analizar la relación que existe entre el programa institucional de tutorías y la formación de los estudiantes universitarios

\section{Interrogante principal}

¿Qué relación existe entre el programa institucional de tutorías y la formación de los estudiantes universitarios?

\section{Hipótesis}

$\mathrm{Hi}_{1}$ existe relación de impacto entre el programa institucional de tutorías y la formación de los estudiantes universitarios.

\section{Resultados}

\section{Análisis Descriptivo}

\section{Frecuencias y porcentajes}

Del total de la muestra de 100 sujetos encuestados, se observa que la mayoría son mujeres; en cuanto al estado civil la generalidad son solteros, así mismo el 18\% son foráneos.

\section{Análisis Integraciónal}

\section{Factorial}

Para este análisis se trabaja con el procedimiento de comunidades de $\mathrm{r} 2$ con rotación de factores varimax normalizado, nivel de error $p \leq 0.001$ con un nivel de confianza del $99.99 \%$ y un $r \geq$ 0.32 . Se elige el procedimiento comunalidades múltiple $\mathrm{r} 2$ por responder al objetivo general, hipótesis e interrogante principal.
El factor 6. Tutorías y formación de los estudiantes, indica que cuando el programa de servicios personalizados de tutorías otorga apoyo a los alumnos en la toma de decisiones; desarrolla en el alumno la elaboración, el uso de la creatividad, la crítica, el interés por su plan de estudios y el trabajo grupal, le permite utilizar la innovación y los diferentes tipos de material siendo el escrito, didáctico y visual como recursos de aprendizaje.

El factor 8. Tutoría y objetivos planteados, se integra a partir de la tutoría de dirección es decir se le indica al tutorado el camino que lo lleve hacia el propósito señalado acorde a los lineamientos establecidos, lo que provoca en ellos la inventiva y el trabajo en equipo, así mismo contribuye en su visión personal de cumplir con sus objetivos, sus prácticas profesionales y servicios social.

De lo anterior se infiere que el apoyo de servicios personalizado de tutorías tiene impacto en la formación de los estudiantes desde el desarrollo de habilidades como la toma de decisiones y en sus recursos de aprendizaje. Además, en su cumplimiento de objetivos planteados.

\section{Regresión múltiple}

Para establecer un modelo tentativo de explicación del fenómeno de estudio se realizó Análisis de Regresión Múltiple, con un alfa $(\alpha)$ de $\leq 0.05$, con un nivel de confianza del $95 \%$. Este análisis estadístico inicia con la selección múltiple de variables considerando como variable dependiente cumplir con los objetivos Las variables independientes que resultaron predictoras de la variable dependiente son asesoría, dirección, trabajo en equipo y planeación. De lo anterior se infiere que el programa de servicios personalizados de tutorías desde la asesoría y planeación y con trabajo en equipo además de planeación se cumple con los objetivos planteados.

Para optimizar la acción tutorial es importante atender una planeación que indique la programación de actividades que permite la asesoría para la incorporación del trabajo en equipo y así tener el logro de los objetivos planteados. 


\section{Conclusiones}

Con base a los resultados obtenidos de los diferentes niveles estadísticos, se presentan las conclusiones que muestran la perspectiva de: El Programa Institucional de Tutorías (PIT): Impacto en la formación de los estudiantes Universitarios. Se logró responder la interrogante principal ¿Qué relación existe entre el programa institucional de tutorías y la formación de los estudiantes universitarios? en los datos obtenidos se observa que el servicio personalizado a través de la tutoría permite potencializar la formación de los estudiantes que impacta en los objetivos planteados ara dar respuesta a los objetivos y las hipótesis de trabajo se concluye que a través del programa de servicios personalizados de tutorías, así como el apoyo y orientación que ofrece, puede desarrollar diferentes habilidades en el alumno de acuerdo al tipo de intervención que se le otorgue: cuando se siente parte de estas acciones repercute en su desarrollando, como, en su toma de decisiones, en la crítica, la creatividad, el trabajo grupal, y promueve la innovación didáctica escrito y visual como recursos de aprendizaje. La tutoría permite indicar al alumno el camino para atender lo planteado, para de objetivos acorde a los lineamientos establecidos. Esto contribuye en su visión personal de cumplir con lo establecido, sus prácticas profesionales y servicio social. De lo anterior se denota que el apoyo de servicios personalizado de tutorías tiene impacto en la formación de los estudiantes desde el desarrollo de habilidades como en la toma de decisiones y en sus recursos de aprendizaje, además en su cumplimiento de objetivos planteados invitándolo a un mayor compromiso con su formación académica y personal.

\section{Propuestas}

De acuerdo a lo mencionado en las conclusiones El Programa Institucional de Tutorías PIT en el estudiante Universitario, cabe señalar que es de suma importancia continuar formando a los docentes para atender dicha función y sumar estrategias de acercamiento con los tutorados asignados ya que para el alumno dicho programa es una opción, como no lo es para el docente, quien deberá contemplar su función de tutor bajo una responsabilidad en el apoyo y canalización en su caso, acciones trascendente y de impacto en la vida del estudiante

\section{Referencias}

Álvarez, P. (2014). La función tutorial del profesorado universitario: Una nueva competencia de la labor docente en el contexto del EEES. Revista Portuguesa de Pedagogía, 47(2), 85-106.

Antoni Adell, Marc. 2002. Estrategias para mejorar el rendimiento académico de los adolescentes. Pirámide. España.

ANUIES (2001). Programas Institucionales de Tutoría: Una propuesta de la ANUIES para su organización y funcionamiento en las instituciones de Educación Superior. México: ANUIES.

Arredondo, Martiniano y Ricardo Sánchez Puentes (2004), Campo científico y formación en el posgrado. Procesos y prácticas de las ciencias experimentales en la UNAM, México, CESU-UNAM/Plaza y Valdés.

Bauman, Zygmunt (2003), Modernidad líquida, México, Fondo de Cultura Económica. Clark, Burton (1997), Las universidades modernas. Espacios de investigación $y$ docencia, México, Miguel Ángel Porrúa/UNAM-Coordinación de Humanidades.

Correa, A. J. (2007). Proyecto: Diseño e implementación de estrategias de intervención para el acompañamiento y orientación académica de los estudiantes de la Universidad Nacional de Colombia: Primer Encuentro de Experiencias Significativas en Acompañamiento Académico a los Estudiantes. Bogotá: Universidad Nacional de Colombia.

Escámez, J., \& Sales, R. G. (2002). Claves educativas para escuelas no conflictivas. Barcelona: Idea books.

Hernández Sampieri, Roberto; et al. Metodología de la Investigación. $4 \mathrm{t}^{\mathrm{a}}$. ed. McGraw-Hill. México, D.F., 2014. Pág. 52 134.

León Hernández, V. y Lugo Villaseñor, E. (2008). Los programas institucionales de tutoría en la universidad pública en México: El caso de las universidades públicas estatales de la región Centro-Sur de la ANUIES. Michoacán: Universidad Autónoma del Estado de Morelos. 
Romo López, A. (2014). Los programas institucionales de tutoría: Actores, procesos y contextos. México: ANUIES.

Sánchez Sánchez, G. I. y Jara Amigo, X. E. (2014). Los espacios de tutoría en práctica profesional y sus necesidades de fortalecimiento. Revista Electrónica Actualidades Investigativas en Educación, 14(2), 1-25.

-Cuevas-Salazar, Omar, Vales-García, Javier y Cruz-Medina, Roberto (2012b). "Impacto del Programa de Tutoría en el desempeño académico de los alumnos del Instituto Tecnológico de Sonora". Revista Electrónica de Investigación Educativa (México), vol.14, núm. 1, pp. 106-121 (en línea). Disponible en: http://redie.uabc.mx/vol14no1/contenidogarciaetal.html

Li, G., Lin, M., Liu, C., Johnson, A., Li, Y., \& Boggs, G. R. (2019). What Is the Learning Paradigm? 13 Ideas That Are Transforming the Community College World, 33.

- Bryan, C., \& Clegg, K. (Eds.). (2019). Innovative Assessment in Higher Education: A Handbook for Academic Practitioners. Routledge.

- Coughlan, T., Pitt, R., \& Farrow, R. (2019). Forms of innovation inspired by open educational resources: a post-project analysis. Open Learning: The Journal of Open, Distance and e-Learning, 34(2), 156-175.

- Chiu, P. H. P., \& Corrigan, P. (2019). A study of graduate teaching assistants' self-efficacy in teaching: Fits and starts in the first triennium of teaching. Cogent Education, 6(1), 1579964.

- Li, G., Lin, M., Liu, C., Johnson, A., Li, Y., \& Loyalka, P. (2019). The prevalence of parent teacher interaction in developing countries and its effect on student outcomes. Teaching and Teacher Education, 86, 102878.

Loyalka, P. (2019). The prevalence of parent teacher interaction in developing countries and its effect on student outcomes. Teaching and Teacher Education, 86, 102878
Margarita, RAMOS-JAUBERT, Roció Isabel, GARCIACONTRERAS, Laura Patricia y MENDOZA-BELMARES, Abraham. El Programa Institucional de Tutorías (PIT): impacto en la formación de los estudiantes universitarios. Revista de Políticas Universitarias. 2019 